This item was submitted to Loughborough's Research Repository by the author.

Items in Figshare are protected by copyright, with all rights reserved, unless otherwise indicated.

\title{
Using atomistic simulations to model Cadmium Telluride thin film growth
}

PLEASE CITE THE PUBLISHED VERSION

http://dx.doi.org/10.1088/0953-8984/28/10/105002

PUBLISHER

(C) IOP

VERSION

AM (Accepted Manuscript)

PUBLISHER STATEMENT

This work is made available according to the conditions of the Creative Commons Attribution-NonCommercialNoDerivatives 4.0 International (CC BY-NC-ND 4.0) licence. Full details of this licence are available at: https://creativecommons.org/licenses/by-nc-nd/4.0/

\section{LICENCE}

CC BY-NC-ND 4.0

\section{REPOSITORY RECORD}

Yu, Miao, and Steven D. Kenny. 2016. "Using Atomistic Simulations to Model Cadmium Telluride Thin Film Growth". figshare. https://hdl.handle.net/2134/20699. 


\title{
Using atomistic simulations to model Cadmium
}

\author{
Telluride thin film growth
}

\author{
Miao YU*and Steven D. KENNY ${ }^{\dagger}$ \\ Department of Materials, Loughborough Univerisity, Loughborough, \\ Leicestershire, LE11 3TU, United Kingdom
}

January 15, 2016

\begin{abstract}
Cadmium Telluride (CdTe) is an excellent material for low-cost, high efficiency thin film solar cells. It is important to conduct research on how defects are formed during the growth process, since defects lower the efficiency of solar cells. In this work we use computer simulation to predict the growth of a sputter deposited CdTe thin film. On-the-fly kinetic Monte Carlo technique is used to simulate the CdTe thin film growth on the (111) surfaces. The results show that on the (111) surfaces the growth mechanisms on surfaces which are terminated by $\mathrm{Cd}$ or Te are quite different, regardless of the deposition energy $(0.1 \sim 10 \mathrm{eV})$. On the Te-terminated (111) surface the deposited clusters first form a single mixed species layer, then the Te atoms in the mixed layer moved up to form a new layer. Whilst on the Cd-terminated (111) surface the new $\mathrm{Cd}$ and Te layers are formed at the same time. Such differences are probably caused by stronger bonding between ad-atoms and surface atoms on the $\mathrm{Te}$ layer than on the Cd layer.
\end{abstract}

*M.Yu2@lboro.ac.uk

${ }^{\dagger}$ S.D.Kenny@lboro.ac.uk 
Keywords: Modelling; Cadmium Telluride; Thin film growth

\section{Introduction}

In recent years, worldwide energy consumption has grown and this has given rise to concerns over energy security, whilst energy supply has become a key problem all around the world. While energy demand rises, reserves in coal, oil, natural gas and other non-renewable resources, which are nowadays the main resources for world energy supply, become smaller due to human consumption. [1] Also the usage of traditional non-renewable resources produces large amount of greenhouse gases, such as $\mathrm{CO}_{2}$ and $\mathrm{N}_{2} \mathrm{O}$, which greatly effect the temperature of the Earth. [2] People are seeking ways of using more environment-friendly and renewable resources, such as sunlight, wind, tides, waves and geothermal heat for energy supply. Governments have invested huge amount of money in renewable technologies all around the world, and solar power is one of the most promising renewable energies.

In the UK, the renewables' share of electricity generation was a record $19.4 \%$ in 2014 Q1, up 6.9 percentage points on the share in 2013 Q1 [3] and solar photovoltaics (PVs) have an important role to play in this [4].

Solar PVs is now the third most important renewable energy source in terms of globally installed capacity. In 2013, its capacity increased by 38 percent to a running total of 139 GW worldwide [5]. By far, the most prevalent material for solar cells is crystalline silicon. But thin film PVs devices have great potential and are a cheaper technology than conventional Si based photovoltaic devices [6].

Cadmium Telluride (CdTe) is an excellent material for low-cost, high efficiency thin film solar cells, and it is the only thin film photovoltaic technology to surpass crystalline silicon PVs in the watt/cost measure and have promising efficiency $[7,8]$. However the laboratory record efficiency of CdTe solar cells lags significantly behind the theoretical maximum for the material. This discrepancy is often attributed to defects such as grain boundaries and intra-grain dislocations [9]. Thus it is important 
to do research on how these defects are formed during the growth process and therefore strategies for reducing them.

Atomistic simulation is widely used as an outstanding partner with experiment in addressing problems in material sciences. By changing the parameters in the simulations, we can simulate different experimental methods for producing thin film cells, e.g. magnetron sputtering [10] and close space sublimation [11]. In this work, we use computer simulation to gain understanding and predict the growth of the sputter deposited thin film PVs as a function of the growth conditions.

Molecular dynamics (MD) is one of the atomistic simulation techniques used in material sciences. In this method, an appropriate interatomic potential is chosen to describe the atomic forces, and the motion of atoms can be simulated by solving Newton's equations of motion. One can model the dynamics by integrating the equations of motion numerically. Resolving individual atomic vibrations requires a time step of the order of femtoseconds (fs) to integrate of the equations of motion. Therefore typical MD simulations can only reach the picosecond or nanosecond time scale.

Many important processes that occur over longer time scales, such as defect diffusion and recombination or thin film growth [12], cannot be simulated by MD. The kinetic Monte Carlo (KMC) algorithm, one of the long time scale dynamics techniques, is able to simulate these rare events. Different from MD, KMC doesn't simulate the atomic vibrations within a state. Instead, one transition is chosen from a list of possible transition events, and then the system is advanced to a new state. The clock is incremented in a way that is consistent with the average time for escape from that state.

The KMC algorithm requires a pre-determinate list of all possible diffusion events, this is extremely difficult to do for thin film growth due to the complex growth environment. We use a variation on the KMC method, the on-the-fly KMC (OTF-KMC) [13], for CdTe thin film growth simulations in this work. The OTF- 
KMC algorithm aiming at find all low-lying saddles surround the states, starts searches from randomly placed configurations, thus building a KMC catalogue on the fly.

In this work, we simulated the growth processes of CdTe thin films on (111) CdTe surfaces at the atomistic level. Effort are focused on the growth mechanisms observed in the simulations and quality of produced film under different conditions. [14]

\section{Methodology}

Thin film growth consists of two processes: the deposition process - allowing clusters be deposited onto the surface at time scale of picoseconds, and the diffusion process - allowing the adatoms to diffuse around the surface at time scale varies from nanoseconds to microseconds. In this work, we use MD to simulate the deposition process and use the OTF-KMC to simulate the diffusion process. Combining those two techniques, we are able to simulate the growth process which occur over time scale of seconds.

In this work, the MD code we are using for the simulations is the LAMMPS package (Large-scale Atomic/Molecular Massively Parallel Simulator [15, 16]), an open source code using classical MD. We use analytical bond-order potentials (BOPs) [17, 18] for the CdTe binary system [19, 20] as the interatomic potential. The BOPs are based upon quantum-mechanical theories and can offer a more accurate description of interatomic interactions compared to Tersoff [21] and Brenner [22] types of potentials. The Tersoff and Brenner types of potentials only consider the $\sigma$ bonding with a second-moment approximation, while the BOP incorporating both $\sigma$ and $\pi$ bondings with a more advanced four-moment approximation.

The OTF-KMC code used in this work is the LKMC (Loughborough on-thefly Kinetic Monte Carlo [23]), which implements an atomistic, multi time scale technique incorporating MD and OTF-KMC. The implementation of OTF-KMC includes transition reuse, a superbasin method for dealing with the low barrier problem 
and an on-the-fly calculation of the Vineyard prefactor. The program also includes an external event driver which can be used to perform particle depositions within the KMC framework.

We model 6 double-layers of atoms, with a total 960 atoms for the (111) surfaces. The bottom double-layer is fixed to avoid shifting of the whole lattice due to energetic impacts, and the next double-layer is thermalised during the deposition process to allow the impact energy to dissipate. Periodic boundary conditions are applied in all directions barring the surface normal, and the free boundary condition is applied in the direction of surface normal. The cluster is deposited from approximately $10 \AA$ above the surface.

We have deposited $\mathrm{Cd}_{x} \mathrm{Te}_{y}(x, y=0,1)$ clusters onto a perfect CdTe surface with various conditions such as substrate, temperature, deposition energy and growth rate. The deposited clusters are allowed to diffuse on the surface. With more and more clusters deposited onto the surface new layers are formed and the CdTe thin film grows.

The growth conditions vary for different experimental techniques. We model the growth of CdTe thin films by magnetron sputtering deposition, which is a widely used technique for CdTe thin film production. In sputtering room temperature deposition is the most common, but elevated temperatures can be used to optimise performance [24]. The temperature we used in the simulations is $T=350 \mathrm{~K}$, all particles are deposited normal to the surface.

Experimental results show that single phase CdTe can only support a maximum excess of 0.004 at $\%$ of $\mathrm{Cd}$ or 0.013 at \% of Te [25]. Experiments undertaken by C. Ferekides et al. [26] also indicate that even if there is an excess of $\mathrm{Cd}$, the produced film will always be stoichiometric. In their experiments, while changing the ratio $\mathrm{Cd} / \mathrm{Te}$ in the vapour from 1.0 to 1.3 , the ratio in the produced film is between 0.97 and 1.03. This means the ratio of $\mathrm{Cd}$ and $\mathrm{Te}$ in the vapour has little effect on the stoichiometry of the produced film. For a start, we choose the CdTe molecules as 
the deposited cluster.

The CdTe impact energy for magnetron sputtering varies. An earlier study of single molecule depositions by M. Yu et al. [27] show that the higher the energy is, the more defects are formed. Therefore we use low deposition energies in our simulations. We set up several simulations with various deposition energies of $0.1 \mathrm{eV}$, $1 \mathrm{eV}$ and $10 \mathrm{eV}$ per cluster.

Typical deposition rates for sputtering are between $0.1 \mathrm{~nm} / \mathrm{s}$ and $1 \mathrm{~nm} / \mathrm{s}$ [24]. In our simulations, the deposition rate is set to be 10 double-layers $/ \mathrm{s}(\sim 2 \mathrm{~nm} / \mathrm{s})$. The chosen deposition rate is slightly higher than the experimental results because we want to accelerate the simulations by depositing more frequently, thus get new layers faster, as this represents the slowest deposition rate that allows us to deposit a reasonable number of layers. In our simulations, there are 160 atoms per doublelayer on the (111) surfaces and the deposited clusters are CdTe molecules, thus the rate for deposition is $R_{\text {depo }}=800 \mathrm{~s}^{-1}$ for the CdTe clusters.

We simulate the growth of CdTe in the zinc-blende structure, the lowest energy polymorph of CdTe, with a lattice constant of $6.83 \AA$, which is the optimal lattice constant for the BOP. We have concentrated on modelling the commonly observed (111) surfaces.

\section{Results}

In the laboratory, the CdTe layer is usually deposited on the CdS, which has the structure of Wurtzite. The (111) surface of CdS has a hexagonal shape, which is the same as zinc-blende structured (111) surfaces. The main difference between Wurtzite and zinc-blende structures in the [111] direction is the stacking sequences. As known that the stacking sequence of Wurtzite structure is AaBbAaBb, while the stacking sequence of zinc-blende is $\mathrm{AaBbCcAaBbCc}$. In other words, there are "missing" layers in the Wurtzite (111) surfaces compare to the zinc-blende (111) surfaces. Therefore in theory, the CdTe would grow on the (111) plane. However 


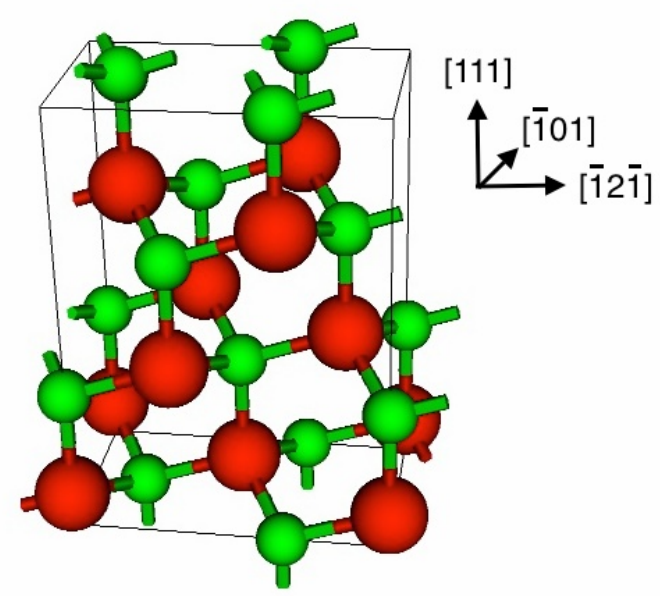

Figure 1 Unit cell of zinc-blende CdTe in [111] direction. Bigger red spheres represent the Te atoms, and smaller green spheres the $\mathrm{Cd}$ atoms.

due to the lattice mismatch, there are other types of surface. Experimental results show that (100) and (111) surfaces are the most commonly observed surfaces for the CdTe layer. A unit cell of zinc-blende CdTe is shown in Figure 1. In the [111] direction, the lattice consists of a Cd layer followed a Te layer (we call both layers together a double-layer).

The results show that single CdTe clusters are quite mobile on both $\mathrm{Cd}$ - and Te-terminated surfaces and they may diffuse hundreds or even thousands of KMC steps before another deposition occurs. We, therefore, start the simulations with 3 deposited CdTe clusters on the Te-terminated (111) surfaces and 4 CdTe clusters on the Cd-terminated (111) surfaces. The isolated CdTe clusters diffuse around and join to become bigger clusters which are less mobile and another deposition is more likely to occur. The OTF-KMC method is used to determine whether to perform a diffusion or a deposition at each step. If a deposition event is selected, the algorithm will switch to MD to perform a deposition.

The growth simulations are undertaken on Cd-terminated (111) surfaces and Te-terminated (111) surfaces. On each surface, we set up several simulations with deposition energies at $0.1 \mathrm{eV}, 1 \mathrm{eV}$ and $10 \mathrm{eV}$. Starting with several ad-atoms deposited on the surface, the OTF-KMC algorithm is used to determine whether to perform a step of diffusion or deposition. 
Despite different deposition energies, all simulations show similar film growth mechanisms for each kind of surface. The results show that single atoms and small ad-atom clusters are highly mobile on the surface. Single Cd/Te atoms or small CdTe clusters diffuse rapidly on the surface until they meet other clusters and form a bigger cluster, which is less mobile. The diffusivity is significantly reduced when the clusters contain at least 4 atoms, as we do not observe any movements involving more than four atoms.

The Cd- and Te-terminated (111) surfaces have different ways of growth, and will now be discussed separately.

\subsection{Te-terminated (111) Surfaces}

On the Te-terminated (111) surfaces, the deposited CdTe clusters usually stick on the surface without dissociation, thus clusters of ad-atoms on the surface are stoichiometric. When the CdTe clusters are deposited onto the surface, they usually "lie down" on the Te-terminated (111) surfaces, where both the Cd and Te atoms are at the same height. The top view and front view of a deposited cluster deposited on the Te-terminated (111) surface are shown in Figure 2.

Bigger clusters are less mobile, and they usually form a zig-zag chain like shape on the surface. These zig-zag clusters are, again, "lying down" on the surface. A top view and front view of zig-zag chains observed in the simulations are shown in Figure 3.

Due to the structure of CdTe lattice, the new CdTe surface layers should grow in pairs in [111] direction, i.e. a new layer of $\mathrm{Cd}$ and a new layer of Te should be formed together (we call both layers together a double-layer). However, on the Te-terminated (111) surfaces, initially when there are not enough ad-atoms on the surface, all deposited ad-atoms are "lying down" on the surfaces, i.e. we have a mixed layer containing both $\mathrm{Cd}$ and Te atoms. We observe zig-zag chains of bigger size on the surface, e.g. the highlighted cluster in Figure 4a. Later when the 


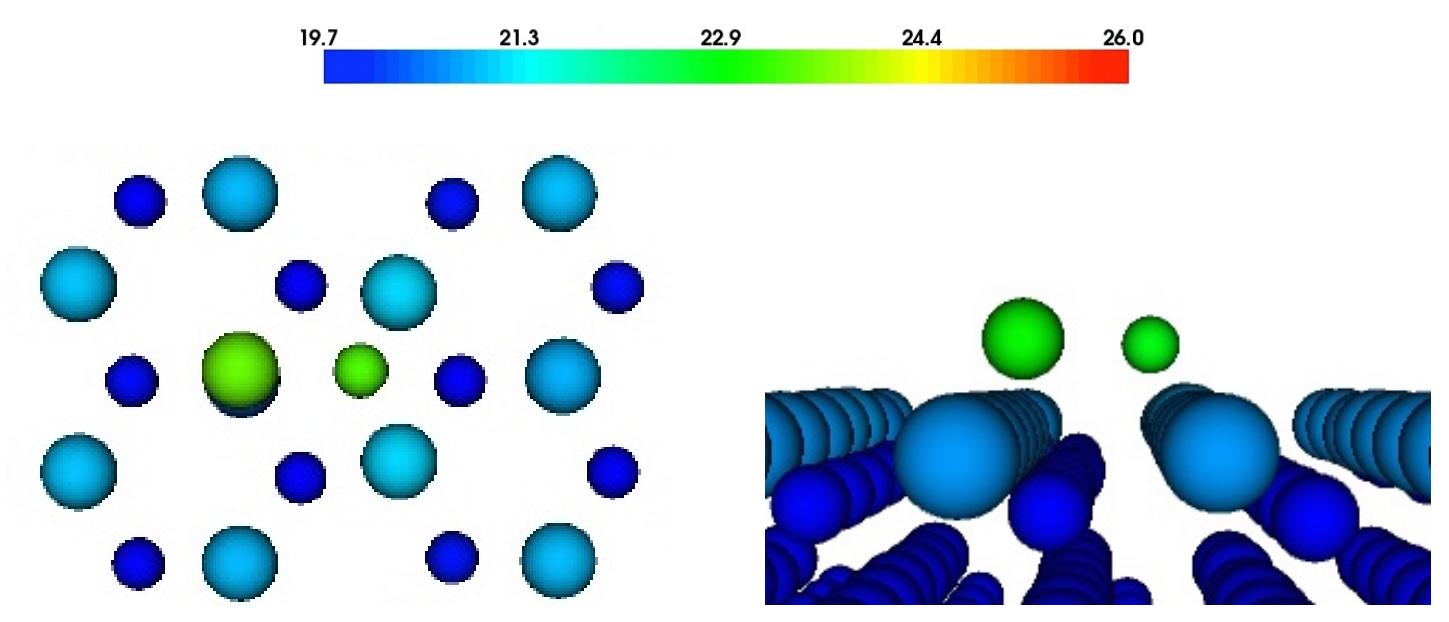

Figure 2 Top view (left) and front view (right) of a CdTe cluster deposited on the Te-terminated (111) surface. The deposited cluster "lies down" on the surface, i.e. both atoms are at the same height. The Te atom sit directly on the surface Te atom, and the $\mathrm{Cd}$ atom sits in the hollow site. Graphs are coloured by height. Bigger spheres represent the Te atoms, and smaller spheres the Cd atoms.

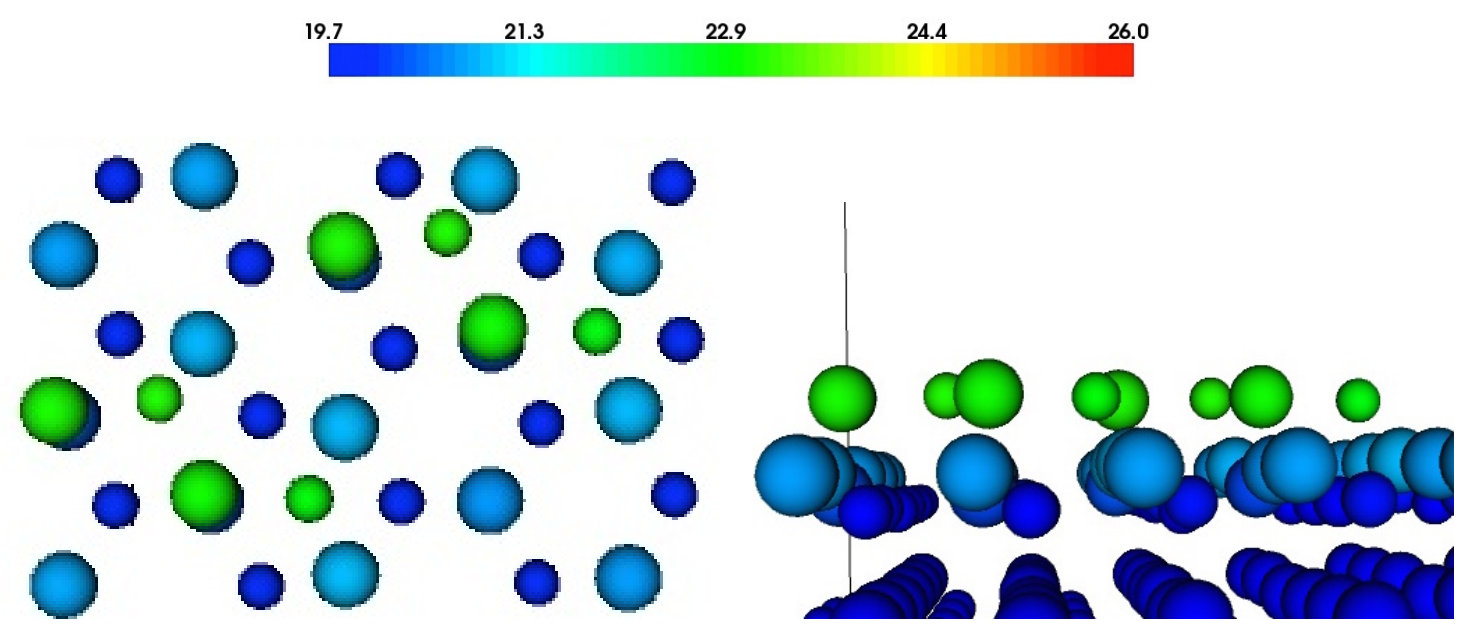

Figure 3 Top view (left) and front view (right) of zig-zag chain shape clusters on the Te-terminated (111) surface. All ad-atoms in the cluster "lie down" on the surface, i.e. they are at the same height. Graphs are coloured by height. Bigger spheres represent the Te atoms, and smaller spheres the Cd atoms. 


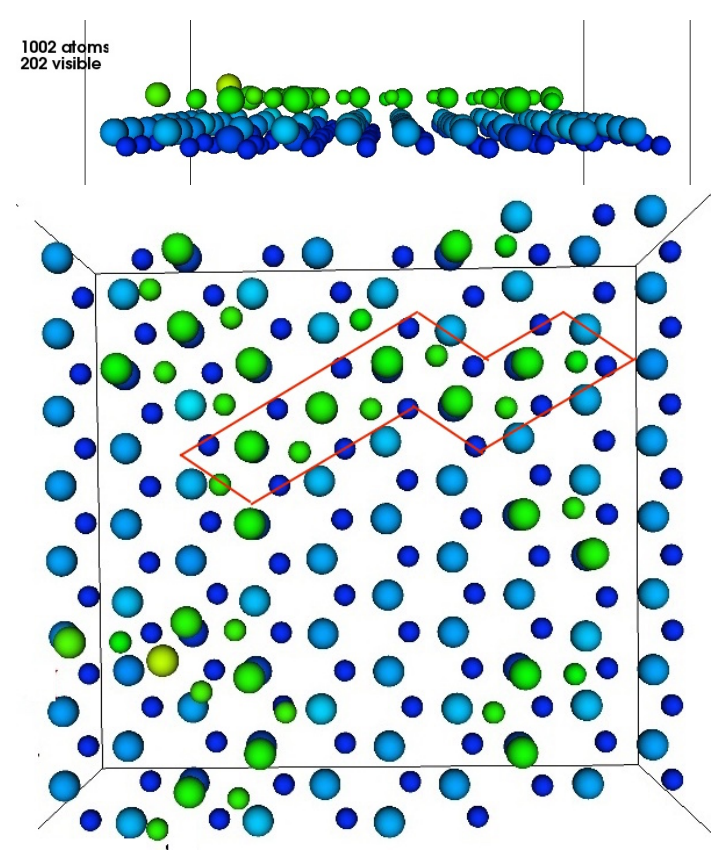

(a) A system with 42 ad-atoms on the Teterminated (111) surface. All ad-atoms stay in the same layer. A cluster of zigzag shape is highlighted.
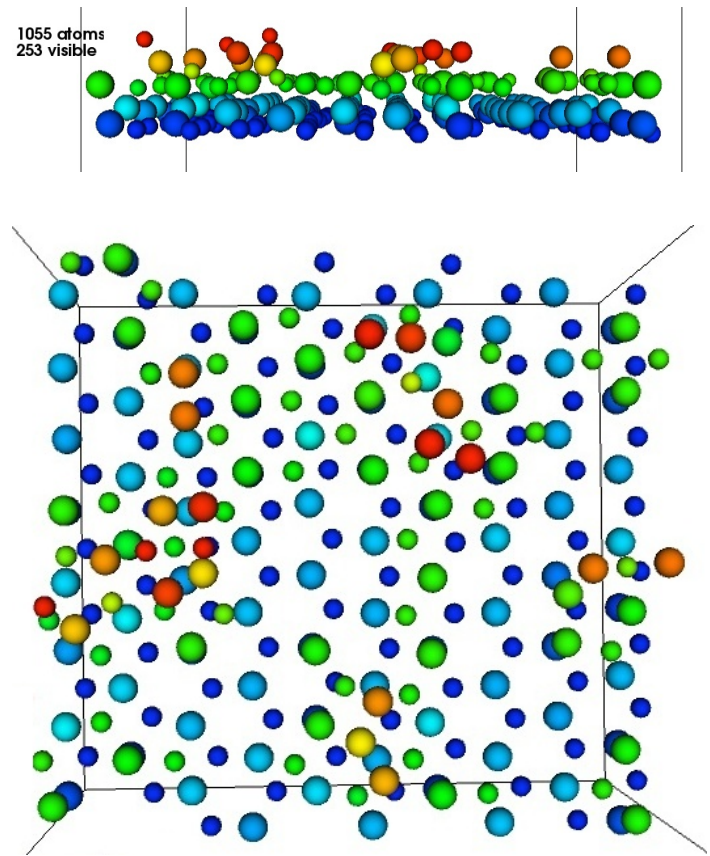

(b) A system with 95 ad-atoms on the Teterminated (111) surface. The second new layer starts to be formed.

Figure 4 Front (upper) and top (lower) views of systems without (left) and with (right) the second layer formed on the Te-terminated (111) surface. The deposited ad-atoms initially "lying down" on the surface. Later with more adatoms are deposited on the surface, bigger clusters would push $\mathrm{Te} / \mathrm{Cd}$ atoms out of the initial layer to form a new layer. Graphs are coloured by height. Bigger spheres represent the Te atoms, and smaller spheres the Cd atoms.

deposited clusters become bigger, a novel mechanism of second layer formation has been observed where the Te atoms move out of the first new layer to form another new layer. These atoms are highly mobile when they move to this new layer and they dominate the dynamics of the system. Figure 4 shows a front and top views of systems without and with the second layer formed. The mechanism of second layer formation will be discussed later.

Since all simulations are undertaken at the same temperature, and the deposition energy is not high enough to damage the original surface, the diffusions of ad-atoms on the surface are quite similar. The main differences among simulations with different deposition energies are observed during the deposition process. Low deposition 


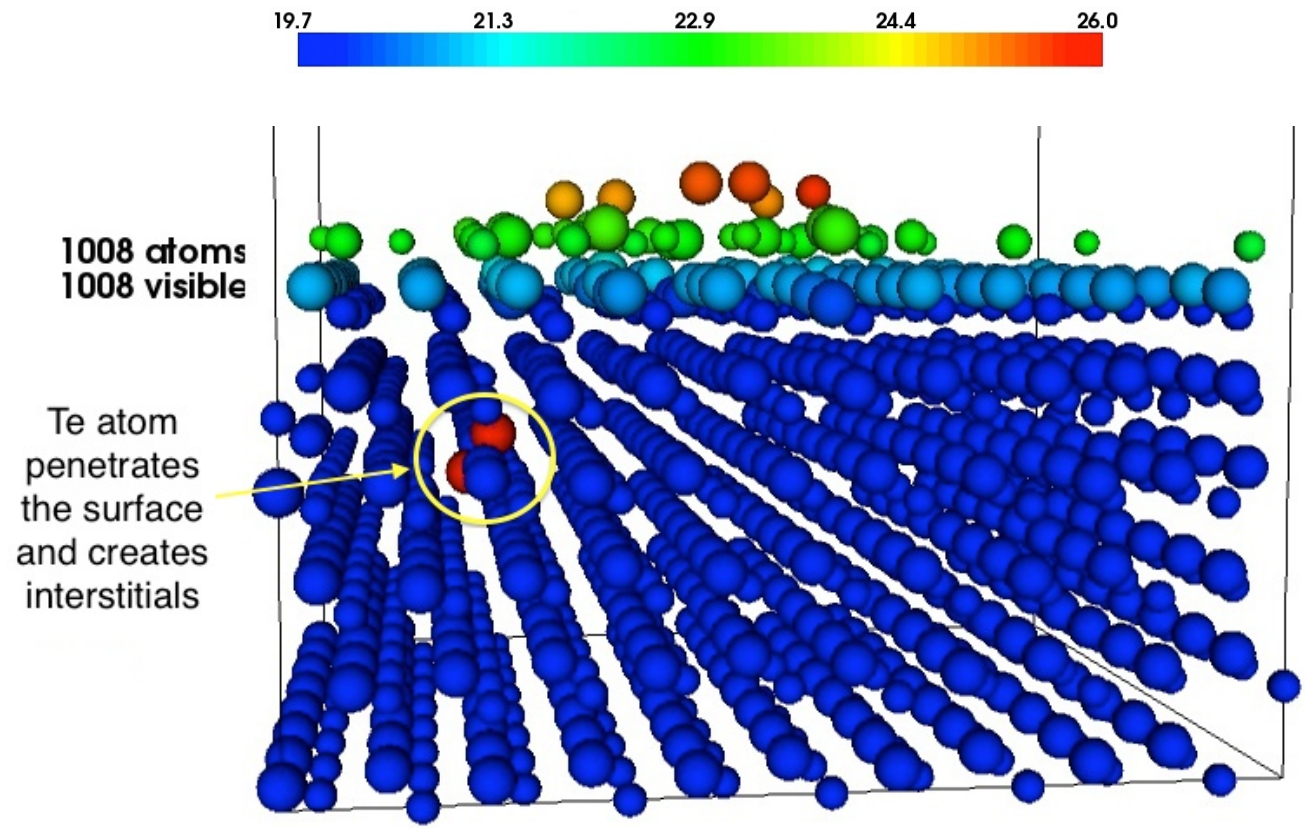

Figure 5 Deposited cluster with high energy penetrates the surface and create interstitials below the surface layers. Graph is coloured by height with highlighting the interstitials below the surface. The Te atom penetrates the Te-terminated surface with deposition energy of $10 \mathrm{eV}$, and creates interstitials $\sim 8 \AA$ below the original surface layer. Bigger spheres represent the $\mathrm{Te}$ atoms, and smaller spheres the $\mathrm{Cd}$ atoms.

energies $(0.1 \mathrm{eV}$ and $1 \mathrm{eV})$ give no significant differences on the depositions. The deposited clusters perform soft landing on the surfaces. However with the higher deposition energy $(10 \mathrm{eV})$, we observe that the deposited cluster may penetrate the surface, and create interstitials below the surface layers. Figure 5 highlights the interstitials created due to the higher deposition energy. The penetrated Te atom creates a Te dimer, and the dimer rotates from time to time during the simulation process.

Figure 6 shows the CdTe thin film growth on Te-terminated (111) surface after $83 \mathrm{~ms}$ of real time. The temperature is $350 \mathrm{~K}$ and the deposition energy is $1 \mathrm{eV}$. There are point defects created on the original surface due to the energetic impacts. Two incomplete new double-layers are formed. In the first new double-layer, most Te atoms still at the same height as $\mathrm{Cd}$ atoms. However in the regions where the second new double-layer is formed, Te atoms in the first new double-layer are 


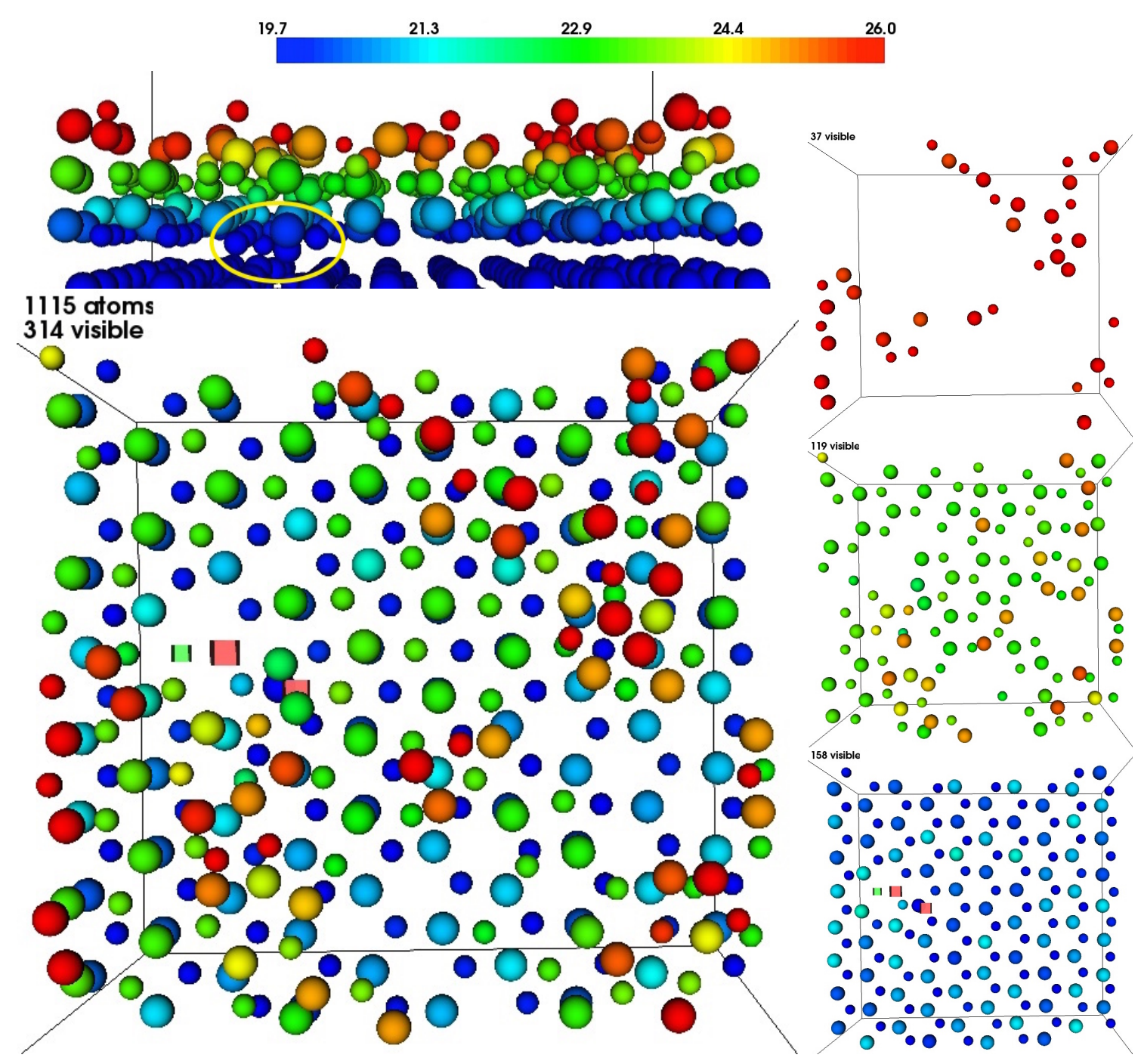

Figure 6 The result of CdTe thin film growth simulation on Te-terminated (111) surface after $83 \mathrm{~ms}$ of real time. The deposition energy is $1 \mathrm{eV}$. A front view (upper left) and a top view (lower left) of the surface and new doublelayers are presented on the left. Three graphs on the right shows the second new double-layer, first new double-layer and the original surface double-layer, respectively. There are 155 ad-atoms on the surface. The circled region in the front view (upper left) of the lattice indicates point defects created due to the energetic impacts. The first new double-layer is incomplete while the second new double-layer starts to form. Most Te atoms in the first new double-layer have the same height as $\mathrm{Cd}$ atoms. Graphs are coloured by height. Green and red cubes represent $\mathrm{Cd}$ and $\mathrm{Te}$ vacancies, respectively. Bigger spheres represent the Te atoms, and smaller spheres the $\mathrm{Cd}$ atoms. 
generally higher than Cd atoms. This indicates that the Te atoms in the first new double-layer will move to their correct heights when the second new double-layer starts to form above them.

Figure 4a shows all deposited clusters stay in the same layer. When a cluster of size 4 or more atoms formed, there is a novel mechanism where the Te atoms move out of the first layer and form a new layer above it. Figure 7 illustrates a typical process by which this occurs, two CdTe clusters diffuse to be adjacent to each other, first three images, and then as indicated by the red shading the two Te atoms diffuse to form a second new layer. This process has a timescale of the order of 200 ps, which is considerably faster than the rate of deposition. The Te atoms are "pushed" onto the second new layer are very mobile as shown in Figure 8. The barrier is lower than most of other transitions, and the corresponding time is much shorter than most of second layer formation transitions and cluster diffusions. Even when the second layer is formed, if there are not enough $\mathrm{Cd}$ atoms in the first layer to "support" them, Te atoms in the second layer can also "drop back" to the first layer. And of course the back-to-first-layer Te atoms can be "pushed" again to the second layer. Figure 9 shows an example of these mechanisms, the mechanisms for the formation and dissolution of the second layer have very similar timescales.

We noticed that, by combining transitions shown in Figure 7 and Figure 9a, the shape of deposited cluster can be changed from zig-zag chain to square shape. We also observe other more complex mechanisms which transform the deposited clusters to other shapes, such as zig-zag chains and hexagons. These indicate that the shape of deposited clusters could be changed.

\subsection{Cd-terminated (111) Surfaces}

The growth mechanism on the Cd-terminated (111) surfaces is different from the Te-terminated ones. One main difference is that the deposited CdTe clusters can dissociate on impacts, thus there are isolated single $\mathrm{Cd} / \mathrm{Te}$ atoms on the surface 


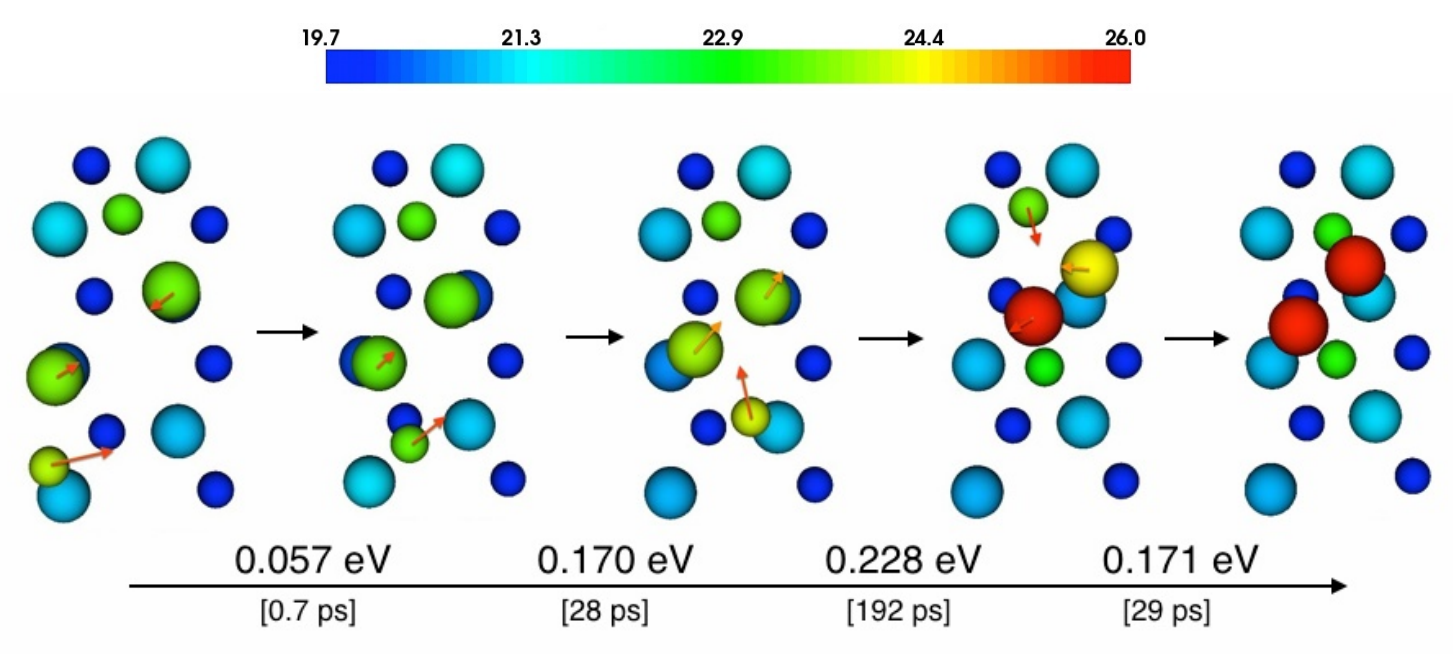

Figure 7 Mechanism of second layer formation in a cluster of 4 atoms. When two CdTe clusters meet each other, the Te atoms in the middle could be "pushed" out of the first layer to form a new layer. Graphs are coloured by height. Bigger spheres represent the Te atoms, and smaller spheres the $\mathrm{Cd}$ atoms. Arrows in the graphs indicate the movement of atoms. The corresponding barriers and event time are given below the graphs.

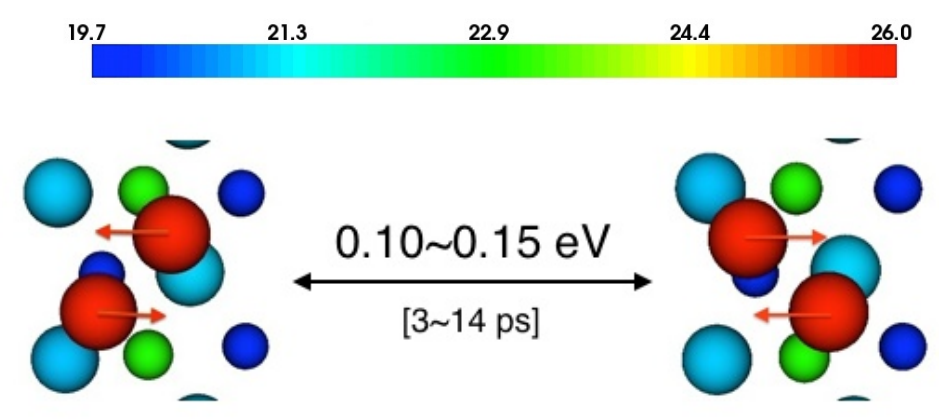

Figure 8 Te atoms in the second new layer are very mobile. Graphs are coloured by height. Bigger spheres represent the Te atoms, and smaller spheres the $\mathrm{Cd}$ atoms. Arrows in the graphs indicate the movement of atoms. 


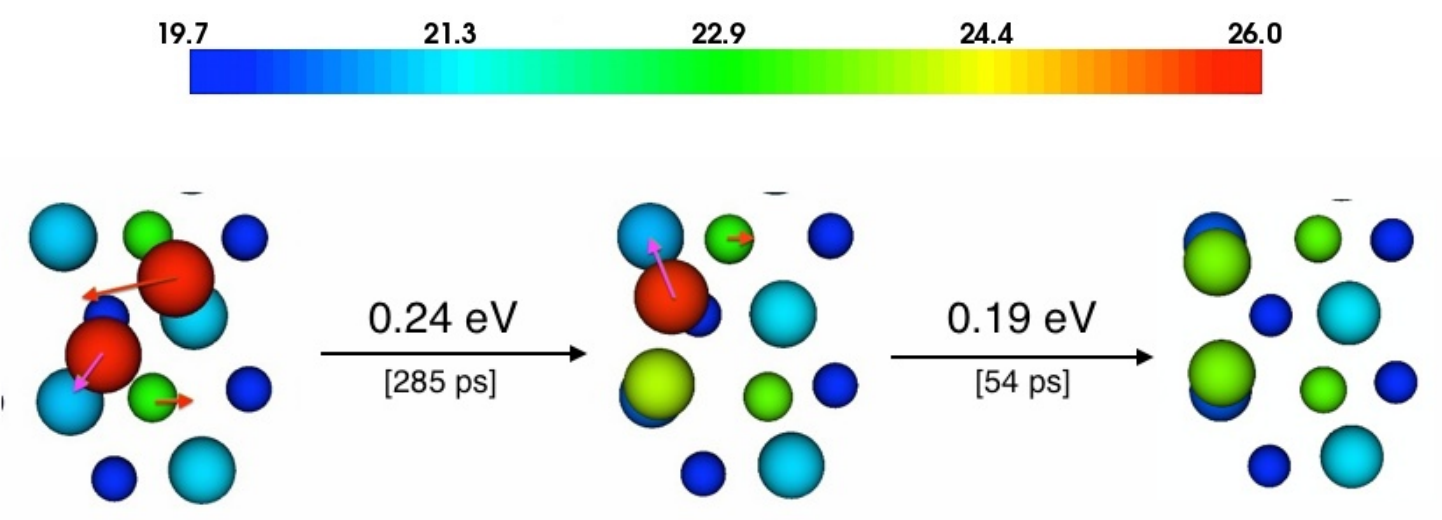

(a) Two Te atoms in the second layer are "pulled" back to the first layer.

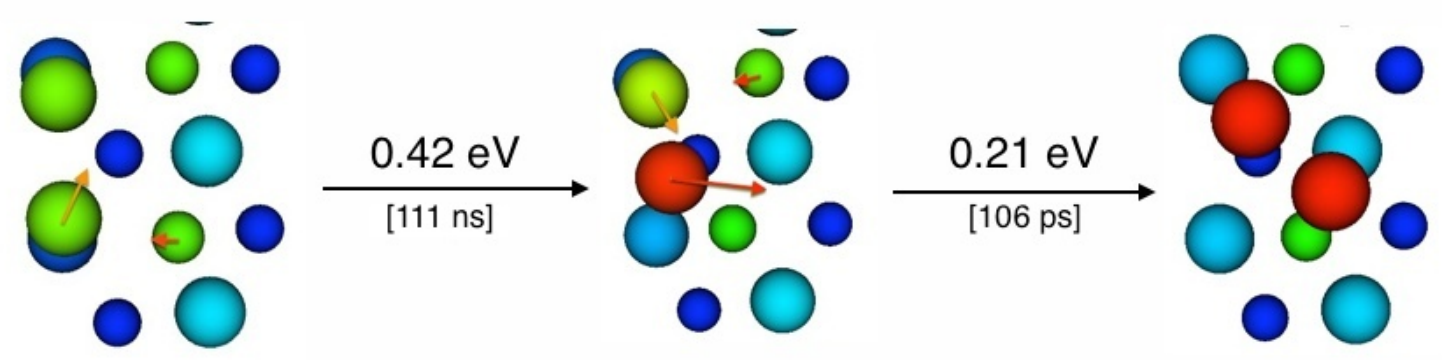

(b) Two Te atoms in the first layer are "pushed" onto the second layer.

Figure 9 An example of Te atoms diffusing between first and second new layers. Graphs are coloured by height. Bigger spheres represent the Te atoms, and smaller spheres the Cd atoms. Arrows in the graphs indicate the movement of atoms. 


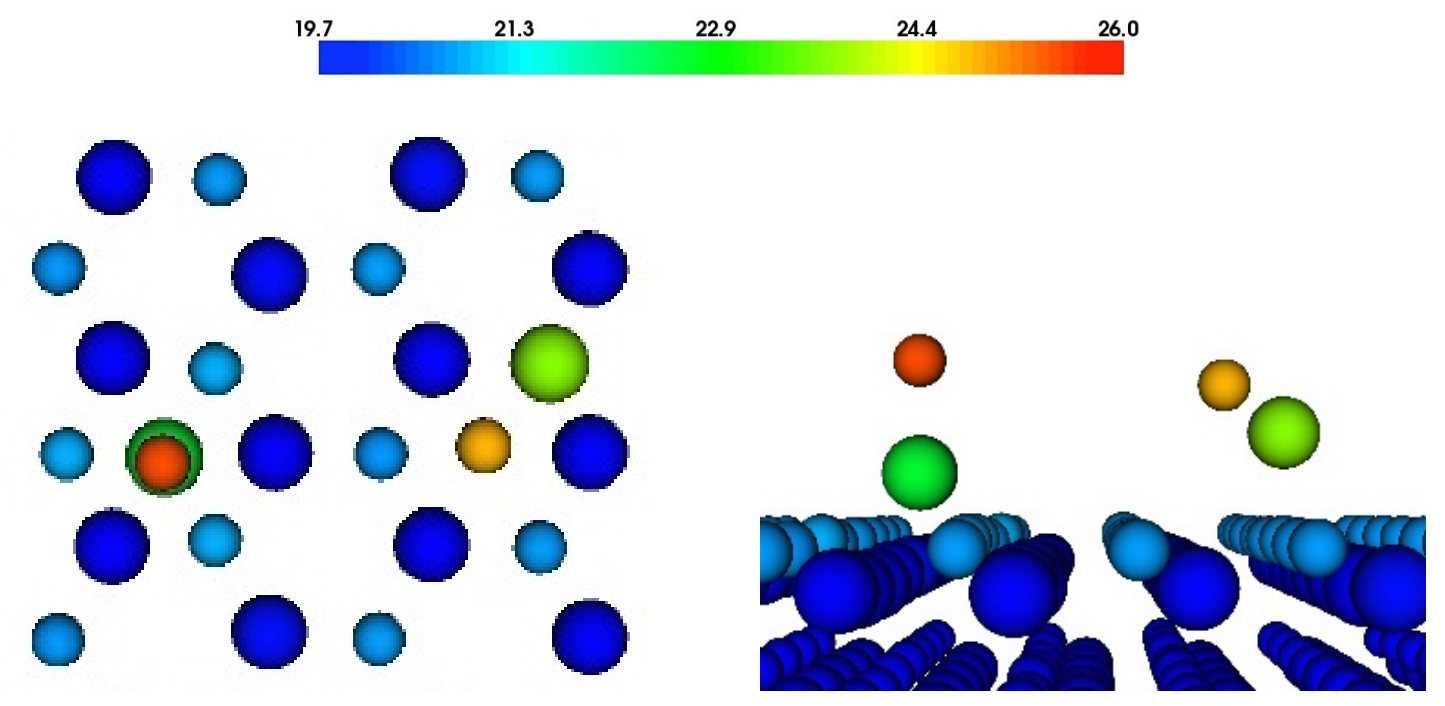

Figure 10 Top view (left) and front view (right) of a small CdTe cluster deposited on the Cd-terminated (111) surface. The deposited clusters "stand up" on the surface, i.e. Cd and Te atoms are at different heights. There are two positions for a single CdTe cluster sits on the surface: (cluster on the left) the CdTe cluster sits vertically in the hollow site; (cluster on the right) Te atom sits directly above the surface $\mathrm{Cd}$ atoms, and $\mathrm{Cd}$ atom in the cluster sits in the hollow site with a higher position. Graphs are coloured by height. Bigger spheres represent the Te atoms, and smaller spheres the Cd atoms.

and the clusters of ad-atoms can be non-stoichiometric. Another main difference is that the CdTe clusters deposited on the Cd-terminated (111) surfaces "stand up", i.e. the $\mathrm{Cd}$ and $\mathrm{Te}$ atoms are at different heights as shown in Figure 10. The new double-layer is formed immediately, no second layer formation is needed.

Similarly, once a big cluster with more than 4 atoms formed, there is usually no movement for the cluster. However, unlike zig-zag shape clusters on the Teterminated (111) surfaces, the ad-atom clusters on the Cd-terminated (111) surfaces are usually in straight line shape in the top view as shown in Figure 11.

The growth mechanism on the Cd-terminated (111) surface is simpler than on the Te-terminated ones. During the simulation, small clusters diffuse around the surface and join other clusters to form a bigger one. The ad-atom-clusters usually form (curved) line shape chains, bigger clusters could form either long line shape chains or double line chains as Figure 12 shows. In the simulations, double line chains are observed more often than long single line chains.

The thin film growth processes on (111) Te- and Cd-terminated surfaces both in- 


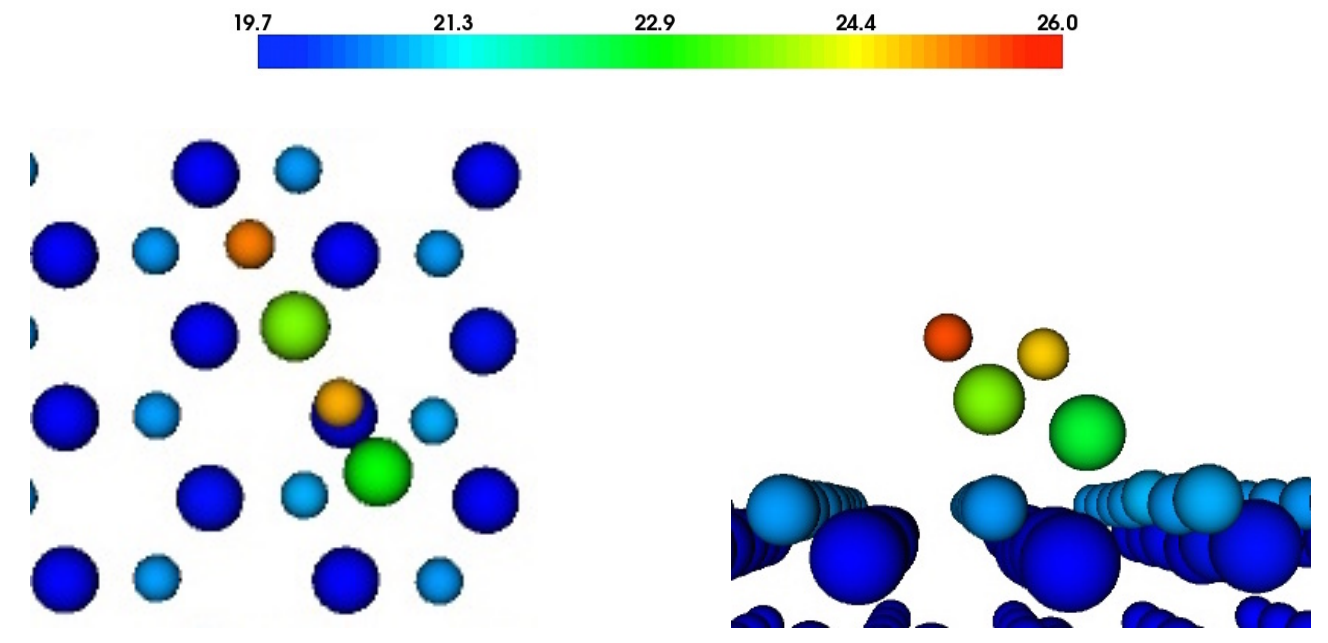

Figure 11 Top view (left) and front view (right) of straight line shape clusters on the Cd-terminated (111) surface. Cd and Te atoms in the cluster are at different heights. Graphs are coloured by height. Bigger spheres represent the Te atoms, and smaller spheres the Cd atoms.

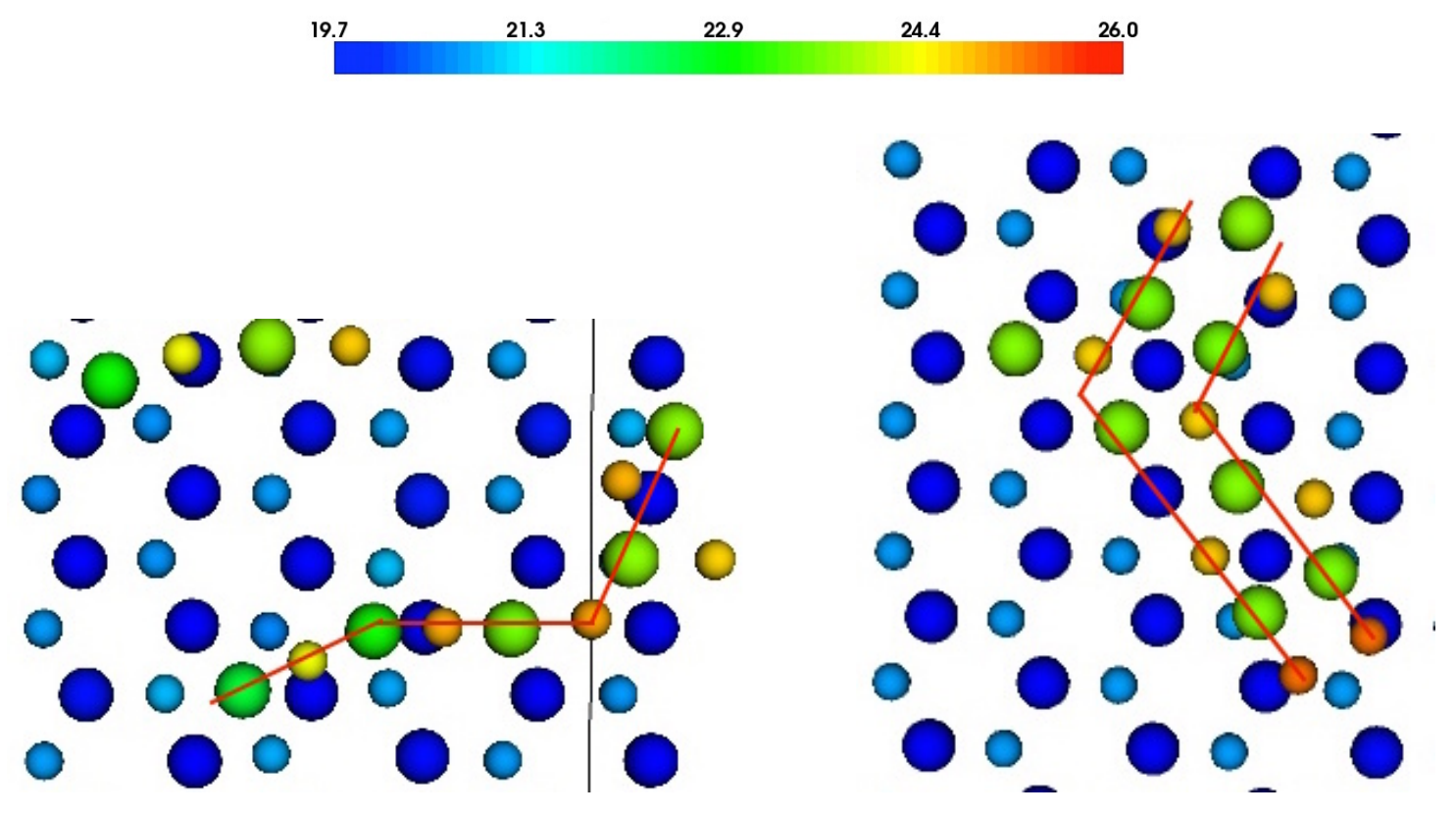

Figure 12 Clusters on the Cd-terminated (111) surfaces form line shape chains. Left: single line chain. Right: double line chain. Graphs are coloured by height. Bigger spheres represent the Te atoms, and smaller spheres the $\mathrm{Cd}$ atoms. 
volve ad-atom-cluster diffusions. We calculated the barriers of a single CdTe cluster diffusion on (111) Te- and Cd-terminated surfaces respectively. When a single CdTe cluster diffuse around the (111) Te-terminated surface, the barriers are ranging from $0.13 \mathrm{eV}[7 \mathrm{ps}]$ to $0.41 \mathrm{eV}[80 \mathrm{~ns}]$ and the most common barriers are around $0.3 \mathrm{eV}$ [2 ns]. However on the (111) Cd-terminated surface, the single CdTe cluster diffusions have much smaller barriers which are ranging from $0.05 \mathrm{eV}[0.5 \mathrm{ps}]$ to $0.3 \mathrm{eV}$ [2 ns]. This is probably because the CdTe cluster on the Te-terminated surface "lies down" on the surface. Both $\mathrm{Cd}$ and Te atoms are very close to the surface atoms and form bonds. While the single CdTe cluster "stands up" on the Cd-terminated surface and only Te atom forms a bond to the surface atoms. It is much easier to break one bond rather than two, thus barriers for single CdTe cluster diffuse around the (111) Cd-terminated surface are much smaller than ones on the Te-terminated surface. This means that the diffusions of small CdTe clusters occur more rapidly on the (111) Cd-terminated surfaces than Te-terminated ones. We even observe the diffusions of a 4-atom-cluster on the (111) Cd-terminated surfaces as shown in Figure 13. The barriers of these transitions are even lower than ones for a single CdTe cluster. This probably because of the structure of 4-atom-cluster where two Cd atoms sit directly above the Te atoms, far away from the surface, therefore the diffusions of this 4-atom-cluster would be similar to the diffusions of a 2-Te-cluster.

The formation of line chains on the CdTe (111) Cd-terminated surface is similar to the growth of $\mathrm{ZnO}$ [28]. As Figure 14 shows that, when a single CdTe cluster diffuse near to other cluster, a longer line shape cluster is formed by attaching the single CdTe cluster to the end of other cluster. Alternately, a "Y" shape cluster is formed by attaching the single CdTe cluster to the middle of other cluster. In most cases, a double line shape cluster is formed by attaching both $\mathrm{Cd}$ and Te atoms in the CdTe cluster to the other cluster as the last graph in Figure 14 shows.

Figure 15 shows the simulation result of CdTe thin film growth on the $\mathrm{Cd}$ terminated (111) surface after $53 \mathrm{~ms}$ of real time. The temperature is $350 \mathrm{~K}$ and 


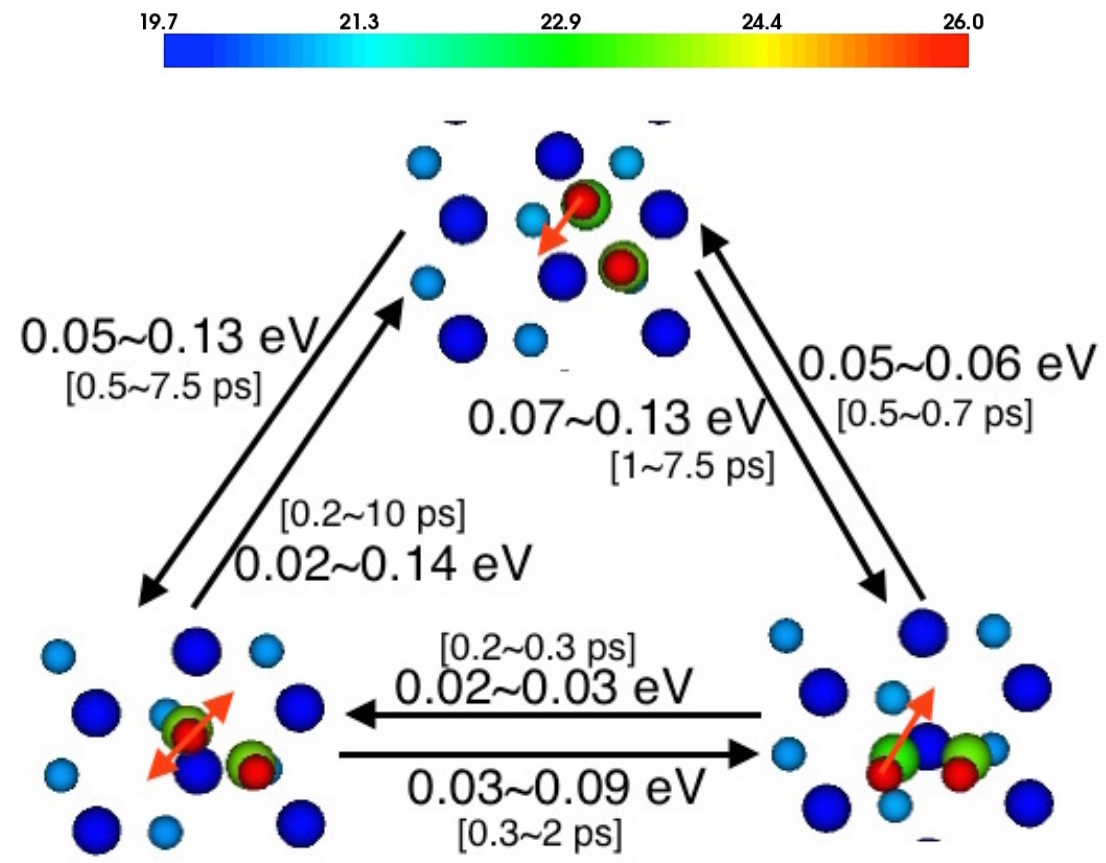

Figure 13 A 4-atom-CdTe cluster diffuses on the Cd-terminated (111) surface. Graphs are coloured by height. Bigger spheres represent the Te atoms and smaller spheres the Cd atoms. Arrows in the graphs indicate the movements of atoms. Square brackets give the corresponding event time of the transition.

the deposition energy is $10 \mathrm{eV}$. We get two incomplete double-layers. There are no defects on the original surface double-layer. The possible defects created during the impacts are recovered due to rapidly diffusions of deposited clusters.

\section{Summary and Conclusions}

We performed simulations of CdTe thin film growth on the (111) Cd- and Teterminated surfaces with various of deposition energies from $0.1 \mathrm{eV}$ to $10 \mathrm{eV}$ at room temperature. The results show that, on the (111) Te-terminated surfaces, the deposited CdTe clusters "lie down" on the surface, i.e. both $\mathrm{Cd}$ and Te atoms are at the same height and they stay in the same layer. The deposited clusters usually in a zig-zag chain shape. In clusters of 4 or more atoms, the Te atoms could move out of the initial layer to form a new layer, therefore forms a double-layer. The atoms being "pushed" to the second layer are so mobile that they may dominate 


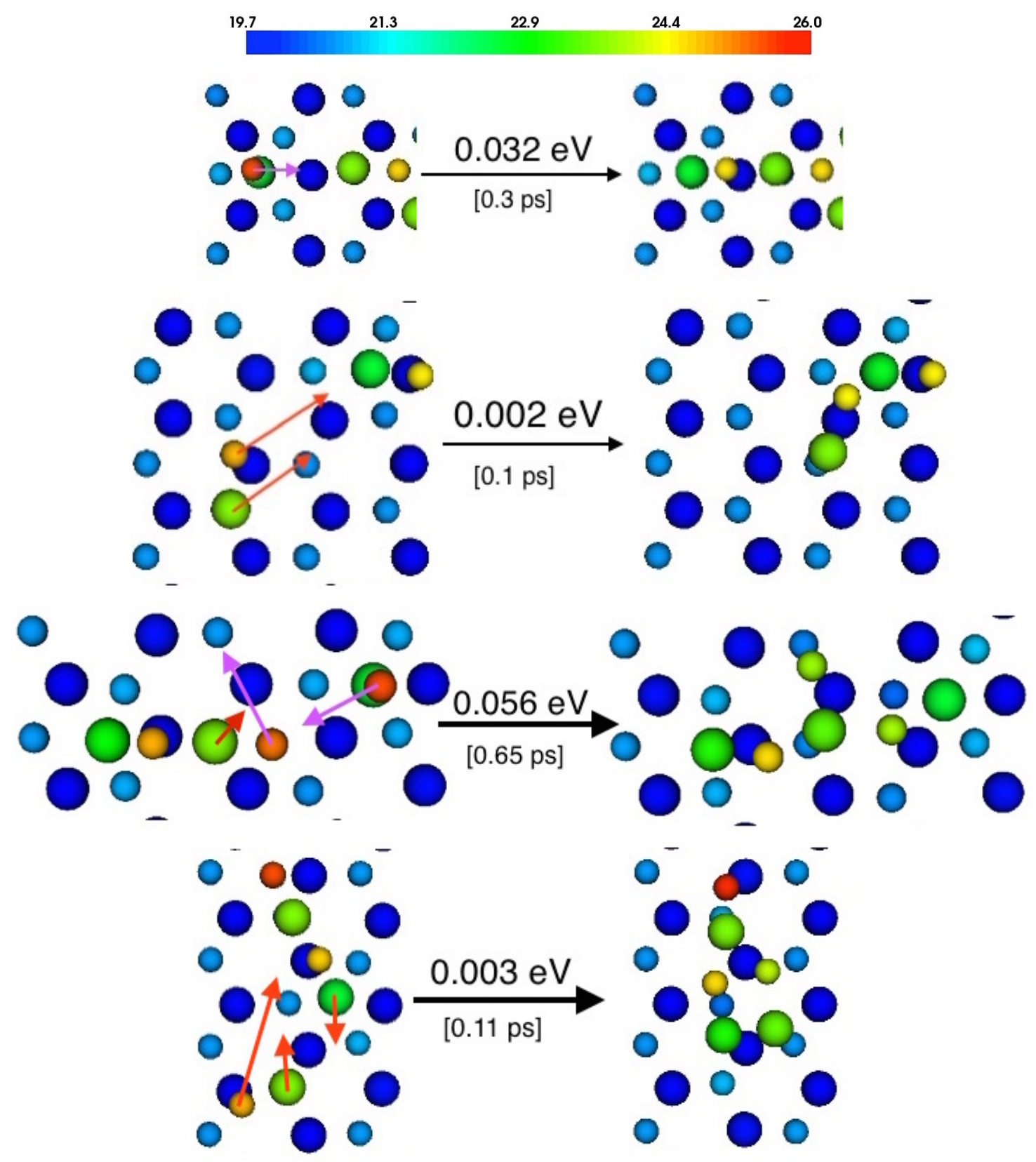

Figure 14 Formation of chains on the Cd-terminated (111) surfaces. U: A single CdTe cluster diffuses to join the end of a chain, and a longer chain is formed. Lower: A single CdTe cluster diffuses near to an end of a chain, and a "Y" shape cluster is formed by attaching the single CdTe cluster to the middle of line chain. Graphs are coloured by height. Bigger spheres represent the Te atoms, and smaller spheres the Cd atoms. Arrows in the graphs indicate the movements of atoms. Square brackets give the corresponding event time of the transition. 


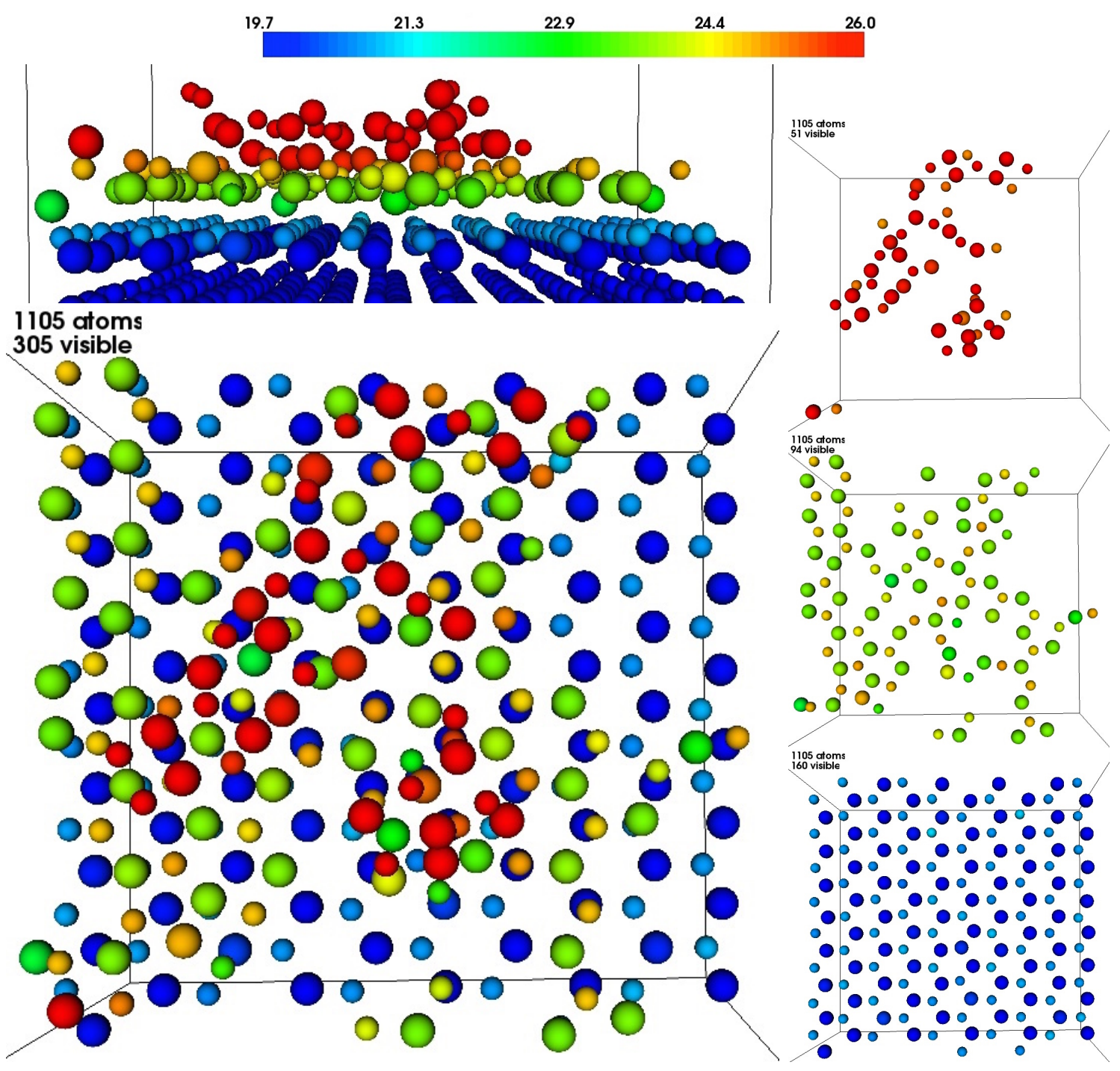

Figure 15 The result of CdTe thin film growth simulation on Cd-terminated (111) surface after $83 \mathrm{~ms}$ of real time. The deposition energy is $10 \mathrm{eV}$. A front view (upper left) and a top view (lower left) of the surface and new doublelayers are presented on the left. Three graphs on the right shows the second new double-layer, first new double-layer and the original surface double-layer, respectively. There are 145 ad-atoms on the surface. The first new doublelayer is incomplete while the second new double-layer starts to form. Graphs are coloured by height. Green and red cubes represent $\mathrm{Cd}$ and Te vacancies, respectively. Bigger spheres represent the Te atoms, and smaller spheres the Cd atoms. 
the simulations. Such atoms in the second layer could also "drop" back to the first layer. The simulation results show that atoms above the double-layer is also helpful to "keep" Te atoms in the first double-layer at their correct heights.

On the (111) Cd-terminated surfaces, the deposited CdTe clusters "stand up" on the surface, i.e. $\mathrm{Cd}$ and $\mathrm{Te}$ atoms are at different heights as expected. The new double-layers are formed with the depositions. Diffusions on the (111) Cdterminated surfaces are more rapidly than on Te-terminated surfaces. We also observe the dissociation of CdTe clusters, which is not seen on the (111) Te-terminated surfaces. Clusters are usually in (double) line chain shapes.

The deposited CdTe cluster usually stick together on the (111) Te-terminated surfaces, while they might dissociate on the Cd-terminated surfaces. Thus the clusters on the (111) Te-terminated surfaces are stoichiometric, but they can be nonstoichiometric on the (111) Cd-terminated surfaces. After the same real time of simulations, there are fewer atoms in the final lattice for the growth simulations on (111) Cd-terminated surface than on Te-terminated surface. This indicates that the growth rate on the Cd-terminated surfaces is lower than the one on Te-terminated surfaces.

Although with deposition energy $10 \mathrm{eV}$ there is a Te atom penetrate the surface and creates interstitials, the CdTe surface is soon recovered within a few KMC steps. We conclude that at room temperature, there is no significant differences on the growth mechanisms with different deposition energy ranging 0.1 10 eV. However the species of surface atoms matters. The Te layers "attract" deposited clusters more than Cd layers, which causing the differences of growth mechanisms on two surfaces. On the Te-terminated surface, a mixed species layer is formed first, then Te atoms move out of the initial layer to form a new layer and a double-layer is formed. On the Cd-terminated surface, the double-layer is formed immediately after the deposition. Diffusions on the (111) Cd-terminated surfaces are more rapidly than on Te-terminated surfaces. 
This work has provided an excellent insight into the CdTe thin film growth with different conditions. It provided a precise understanding of the process during the thin film growth. Specific transitions of the growth mechanisms are studied on each surface to understand how the thin film growths.

\section{A References}

[1] S. Shafiee and E. Topal, "When will fossil fuel reserves be diminished?," Energy policy, vol. 37, pp. 181-189, Jan. 2009.

[2] M. E. Mann, R. S. Bradley, and M. K. Hughes, "Global-scale temperature patterns and climate forcing over the past six centuries," Nature . ., vol. 392, pp. 779-787, Apr. 1998.

[3] DECC, "Energy trends: June 2014," Energy Trends, June 2014. https: //www . gov.uk/government/publications/energy-trends-june-2014.

[4] H. Spanggaard and F. C. Krebs, "A brief history of the development of organic and polymeric photovoltaics," Solar Energy Materials and Solar Cells, 2004.

[5] EPIA, "Global market outlook for photovoltaics 2014-2018," Global Market Outlook for Photovoltaics, June 2014. http://www.epia.org/ fileadmin/user_upload/Publications/EPIA_Global_Market_Outlook_ for_Photovoltaics_2014-2018_-_Medium_Res.pdf.

[6] G. Di Marco and L. Palmisano, "Thin-Film Photovoltaics," International Journal of Photoenergy, vol. 2010, pp. 1-2, 2010.

[7] W. Shockley and H. J. Queisser, "Detailed Balance Limit of Efficiency of p-n Junction Solar Cells," Journal of Applied Physics, vol. 32, no. 3, pp. 510-519, 1961. 
[8] M. A. Green, "Radiative efficiency of state-of-the-art photovoltaic cells," Progress in Photovoltaics: Research and Applications, vol. 20, pp. 472-476, Sept. 2011.

[9] J. Y. W. Seto, "The electrical properties of polycrystalline silicon films," Journal of Applied Physics, vol. 46, no. 12, pp. 5247-5254, 1975.

[10] A. D. Compaan, A. Gupta, S. Lee, S. Wang, and J. Drayton, "High efficiency, magnetron sputtered CdS/CdTe solar cells," Solar Energy, 2004.

[11] S. N. Alamri, "The growth of CdTe thin film by close space sublimation system," physica status solidi (a), vol. 200, pp. 352-360, Dec. 2003.

[12] S. Blackwell, R. Smith, S. D. Kenny, and J. M. Walls, "Modeling evaporation, ion-beam assist, and magnetron sputtering of thin metal films over realistic time scales," Physical Review B, vol. 86, p. 035416, July 2012.

[13] G. Henkelman and H. Jónsson, "Long time scale kinetic Monte Carlo simulations without lattice approximation and predefined event table," The Journal of Chemical Physics, vol. 115, no. 21, p. 9657, 2001.

[14] L. Vernon, S. D. Kenny, R. Smith, and E. Sanville, "Growth mechanisms for TiO2 at its rutile (110) surface," Physical Review B, vol. 83, p. 075412, Feb. 2011.

[15] LAMMPS, "Lammps molecular dynamics simulator." Accessed date, Aug. 2014. http://lammps.sandia.gov/.

[16] S. Plimpton, "Fast Parallel Algorithms for Short-Range Molecular Dynamics," Journal of Computational Physics, 1995.

[17] D. G. Pettifor and I. I. Oleinik, "Bounded analytic bond-order potentials for $\sigma$ and $\pi$ bonds," Physical Review Letters, vol. 84, no. 18, pp. 4124-4127, 2000. 
[18] D. G. Pettifor and I. I. Oleinik, "Analytic bond-order potentials beyond TersoffBrenner. I. Theory," Physical Review B, vol. 59, no. 13, p. 8487, 1999.

[19] D. K. Ward, X. W. Zhou, B. M. Wong, F. P. Doty, and J. A. Zimmerman, "Analytical bond-order potential for the cadmium telluride binary system," Physical Review B, vol. 85, p. 115206, Mar. 2012.

[20] D. K. Ward, X. W. Zhou, B. M. Wong, F. P. Doty, and J. A. Zimmerman, "Accuracy of existing atomic potentials for the CdTe semiconductor compound," The Journal of Chemical Physics, vol. 134, no. 24, p. 244703, 2011.

[21] J. Tersoff, "Modeling solid-state chemistry: Interatomic potentials for multicomponent systems," Physical Review B, vol. 39, pp. 5566-5568, Mar. 1989.

[22] D. W. Brenner, "Empirical potential for hydrocarbons for use in simulating the chemical vapor deposition of diamond films," Physical Review B, vol. 42, no. 15 , p. 9458, 1990.

[23] C. Scott, T. Lazauskas, M. Yu, L. Vernon, and S. D. Kenny, "LKMC. Loughborough on-the-fly Kinetic Monte Carlo," Computer Physics Communications, 2015. Preprint submitted.

[24] J. M. Walls, "Department of Electrical Engineering, Loughborough University, Leicestershire, U.K.." private commun.

[25] J. H. Greenberg, "P-T-X phase equilibrium and vapor pressure scanning of nonstoichiometry in CdTe," Journal of crystal growth, vol. 161, no. 1-4, pp. 1-11, 1996.

[26] C. Ferekides, "Thin film CdTe photovoltaics: Past, present and future," presentation, University of South Florida, USA, Sept. 2014.

[27] M. Yu and S. D. Kenny, "The energetic impact of small CdxTey clusters on Cadmium Telluride," Thin Solid Films, vol. 584, pp. 41-45, June 2015. 
[28] S. Blackwell, R. Smith, S. D. Kenny, J. M. Walls, and C. F. Sanz-Navarro, "Modelling the growth of $\mathrm{ZnO}$ thin films by PVD methods and the effects of post-annealing," Journal of Physics: Condensed Matter, vol. 25, p. 135002, Apr. 2013. 